\title{
Hyperbaric oxygen therapy as a potential therapy for new-onset diabetes mellitus in post-COVID-19 syndrome: current evidence
}

\author{
Mahrumi Dewi Tri Utami ${ }^{1}$, Rezy Ramawan Melbiarta ${ }^{1}$, Zefo Kiyosi \\ Wibowo ${ }^{1}$, Rimbun ${ }^{2, *}$ \\ Email: rimbun@fk.unair.ac.id \\ ${ }^{1}$ Medical Program, Faculty of Medicine, Universitas Airlangga, Surabaya, Indonesia \\ ${ }^{2}$ Department of Anatomy, Histology, and Pharmacology, Faculty of Medicine, Universitas Airlangga, Surabaya, Indonesia
}

\begin{abstract}
New-onset diabetes mellitus (DM) has been lately observed among patients with post-COVID-19 syndrome. ACE2 and TMPRSS22 receptors were found expressed in the pancreas, thus acting as an entry gate leading to infection-induced pancreatic injury. Moreover, COVID-19 and DM have been reported to interact bidirectionally, in which existing DM comorbidity increases the patient's chance of getting severe COVID-19 and vice versa. Hyperbaric oxygen therapy (HBOT) is one of the therapy modalities given by applying administration of oxygen $100 \%$ under pressure of more than 1.4 ATA HBOT has been shown to improve cellular function such as decreasing oxidative stress, inflammation, and mitochondrial dysfunction. These protective effects might correlate with preserved pancreatic cells, which improved insulin homeostasis. However, the potency and mechanism of HBOT to these patients remain unclear. Hence, we conduct a review of the available evidence concerning the potential mechanisms of HBOT in improving new-onset DM among post-COVID-19 syndrome patients. Current literature showed that HBOT might be beneficial for these patients, thus this modality might be a new breakthrough for researchers and health workers considering post-COVID-19 syndrome incidence tends to rise as the pandemic grows.
\end{abstract}

Keywords: Hyperbaric oxygen therapy, Diabetes, COVID 19, Post-acute COVID19 syndrome, SARS-CoV-2

\section{Introduction}

Post-COVID-19 syndrome is syndrome which persist for more than three weeks after the diagnosis of COVID-19. Nearly 10-35\% of COVID-19 patients experienced post-COVID-19 syndrome and this number was higher in hospitalized patients which may reach $85 \%$ [1]. Not only limited to lung manifestation, but the course of the disease might involve various organ systems which later contribute to poorer quality of life $[2,3]$. SARS-CoV-2 expresses S protein that binds to the ACE2 receptors expressed in various organs $[4,5]$. ACE2 receptor was also distributed in pancreatic $\beta$-cells which makes it possible to have a link with the course of diabetes mellitus (DM) $[4,6]$. The emersion of this phenomenon should be taken into consideration as the COVID-19 pandemic still evolves. Furthermore, it was estimated that 280 million people have been confirmed for COVID-19 diagnosis and the number will still grow as the pandemic has not ended yet [7].

Hyperbaric oxygen therapy (HBOT) is a treatment modality in which administers $100 \%$ oxygen in a hyperbaric chamber in 2-3 atmosphere absolute (ATA) which could be carried out in a monoplace or multiple chambers [8,9]. HBOT is commonly indicated for gas embolism, intoxication of carbon monoxide, decompression disease, infections of necrotizing soft tissue, chronic ulcers, ischemic severe multiple traumas, and ischemic diabetic foot ulcers [10]. Despite its effect to improve ischemic diabetic foot ulcers, HBOT has beneficial effects for type 1 and type 2 diabetes mellitus. In the case of T2DM, HBOT could have benefits in 
increasing insulin sensitivity [11]. Blocking of carotid bodies activity in hypoxic conditions is further expected to improve glucose tolerance in T2DM patients as well [12]. HBOT can also contribute to the prevention of T1DM due to its ability to stimulate proliferation and inhibit apoptosis of $\beta$-cell [13].

As mentioned before, a link between COVID-19 and DM has been observed. This would lead to poorer outcomes for the patient concerned. HBOT has potential effects to improve DM experienced by the patients. Moreover, HBOT also might ameliorate lung injury during the course of COVID-19 [14]. Based on this mechanism, HBOT proposes a new modality treatment for new-onset DM in patients with post-COVID-19 syndrome. The aim of this review is to investigate the possible mechanism of HBOT to improve new-onset DM in patients with post-COVID-19 syndrome as it has not been elucidated clearly yet.

\section{Hyperbaric oxygen therapy: mechanism of action}

Hyperbaric oxygen therapy (HBOT) is a treatment modality given to the patient by administering $100 \%$ oxygen inside a hyperbaric chamber under pressure higher than sea level, 1 ATA. HBOT is commonly carried out inside monoplace or multiple chambers and pressurized under 2-3 ATA. Inside monoplace chamber, the entire chamber is filled with $100 \%$ oxygen with a single patient is placed inside the chamber, while multiple chambers can hold for more people with $100 \%$ oxygen is given via individual masks, head hoods, or endotracheal tubes [8,9]. The mechanism of HBOT is in accordance with several gas laws. Dalton's gas law stated that in a mixture of gases, each partial pressure of an individual gas is proportional to its fractional concentration of total volume multiplied by ambient pressure $[9,15]$. It is implied that increased ambient pressure inside the chamber allows higher partial pressure of oxygen to be distributed to the patient [15]. Henry's law stated that in a mixture of liquid or tissue, the sum of gas dissolved matches the partial pressure of the gas. Consequently, an increase in oxygen partial pressure will supply more oxygen to be delivered towards tissue and cell [16]. Most of the oxygen inside the human body was bound by hemoglobin and only a tiny amount was soluble in the plasma. Based on explained laws, oxygen dissolved into the plasma will increase as the concentration and pressure of oxygen breathed are increased [9]. Boyle's law is another law that underlies the indication of HBOT for decompression and emboli disease. In accordance with that law, the volume of a gas bubble is inversely proportional to the exerted pressure [17]. HBOT is commonly indicated for gas embolism, intoxication of carbon monoxide, decompression disease, infections of necrotizing soft tissue, chronic ulcers, ischemic severe multiple traumas, and ischemic diabetic foot ulcers $[10]$.

\section{Hyperbaric oxygen therapy: cellular level mechanism}

Reactive oxygen species (ROS) is a metabolism by-product that commonly produced during hyperoxia conditions. They could be superoxide $\left(\mathrm{O}_{2} \bullet-\right)$, hydrogen peroxide $\left(\mathrm{H}_{2} \mathrm{O}_{2}\right)$, hypochlorous acid (HClO), and hydroxyl (HO•) [18]. Throughout cell metabolism in hyperbaric conditions, ROS is mainly generated with the involvement of mitochondrial respiratory chain (MRC) complexes [17]. RNS (Reactive Nitrogen Species) is another reactive species that contain nitrogen including NO (Nitric Oxide) and other molecules made by reactions between NO with its oxidation products or ROS [9]. Antioxidants, both enzymatic and non-enzymatic, play an important role in scavenging the overproduction of reactive species. Fortunately, current studies showed that exposure to hyperoxia during HBOT is commonly brief enough to let antioxidants preserve the body from the unwanted reactive species stresses and make this effect stay reversible [18]. Previously, RONS (Reactive Oxygen and Nitrogen Species) were only thought of as harmful metabolism by-products. However, now they are known to be a key player in signaling molecules. Therefore, RONS are important molecules to initiate, mediate, and regulate cellular and biochemical oxidative stress and 
can act both as physiological or as pathogenic processes $[9,19]$. The beneficial or detrimental effects of RONS depend on the concentration and intracellular localization [18].

HBOT could induce vascularization by mobilizing stem/progenitor cells (SPCs) through a redoxmediated process and inducing vasculogenesis. This effect was expected to be mediated via upregulation of hypoxia-inducible factor (HIF) transcription factors following HBOT, in which HIF-1 major transcriptional target was vascular endothelial growth factor (VEGF), one of the most vigorous stimulators of neovascularization. Oxidative stress during HBOT also stabilizes the active form of HIF-1. Enhanced the upregulation of Nuclear factor-erythroid-2 related factor 2 (Nrf2) transcription factor expression which is a key antioxidant genes regulator was also seen. So that, antioxidants such as glutathione (GSH), glutamate peroxidase (GPx), and glutamate reductase (GR) expression increased [9,20]. RONS produced during HBOT could react with microbial organelles, lipid, and proteins irreversibly, making it exerts an antimicrobial effect. Moreover, $\mathrm{H}_{2} \mathrm{O}_{2}$ could activate NF- $\mathrm{KB}$, TNF- $\alpha$, and MAPK which manage the expression of growth factors, cytokines, and chemokines, thus acting to enhance immune response [9]. NF- $\mathrm{KB}$ and sirtuin-1 (SIRT1) decrease Bax (pro-apoptosis) expression and together with the increased expression of Bcl-2 (anti-apoptotic) reduce mitochondria-mediated cell death following HBOT [21]. HBOT also modulates inflammation through TNF- $\alpha$ signaling and reduces pro-inflammatory cytokines levels [9].

\section{Hyperbaric oxygen therapy: potency for diabetes mellitus treatment}

As previously mentioned, one of the indications of HBOT is ischemic diabetic foot ulcers. It is explained by the ability of HBOT to ameliorate tissue hypoxia, enhance perfusion, promote fibroblast and collagen production, also induce angiogenesis. Moreover, HBOT has an antimicrobial effect and improves leukocyte and macrophage function, thus it can help to treat infection in soft tissue and bone [9,22]. Diabetic foot is associated with neuropathy and/or peripheral arterial disease that happened as a complication of DM [23]. HBOT is also expected to have a beneficial effect on improving DM itself. Reduction on blood glucose after HBOT was seen in several studies [12,24-29]. A study conducted by Ekanayake and Doolette found that there was no change in insulin secretion level after HBOT was done [24]. Hence, the underlying mechanism of blood glucose reduction was estimated due to increased insulin sensitivity in T2DM [11].

Another possible mechanism is thought to be carotid bodies inactivity during HBOT. Carotid bodies are peripheral sensory chemoreceptors that react to arterial blood. Its sensory activity increases hypoxemia. Carotid bodies also have a sense of arterial blood glucose and circulating insulin levels and are thought to be involved in the development of insulin resistance [11]. In metabolic syndrome and T2DM animal models, abolishment of carotid bodies leads to the prevention of insulin resistance. Hyperoxia conditions in HBOT could block carotid body activity which is further expected to improve glucose tolerance in T2DM [12]. Furthermore, HBOT also contributes to the improvement of T1DM. A study conducted by Faleo et al showed that two-week of HBOT could lead to stimulating proliferation and inhibiting apoptosis of $\beta$-cell [13].

\section{The pathogenesis mechanisms and new-onset diabetes by SARS-CoV-2}

SARS-CoV-2 expresses a $\mathrm{S}$ protein (envelope a protein) that has high affinity to bind the extracellular domain of angiotensin-converting enzyme 2 (ACE2) [30]. SARS-CoV-2 enters host cells through ACE2 which is a functional receptor. Inflammatory changes in lungs such as edema and degeneration occurred because of the invasion virus to the cells [5]. The effect is fusion of the membrane and internalization of the virus by endocytosis [31]. The replication of the virus causes loss of ACE2, thus 
preventing degradation of Angiotensin II into Angiotensin-(1-7) [32]. These conditions might result in COVID-19-associated fibrosis which contributes to lung damage. These changes might lead to lung injury mechanisms, hypoxia by myocyte injury, immune response, myocardial cell damage, intestinal and cardiopulmonary issues [5].

This mechanism should be taken into consideration because the pancreas expresses ACE2 as well, so it can manifest to pancreatic injury [33-35]. In severe cases, pancreatic injury can be a serious problem due to its impact on the quality of life of the patients. The serious effects of SARS-CoV-2 include systemic inflammation, acceleration of acute respiratory distress syndrome, and the development of chronic pancreatitis [33]. Patients might present with abdominal pain and an increase of serum amylase or lipase which indicate exocrine pancreatic injury [36]. The other mechanism of SARS-CoV-2 infection is the occurrence of immune reaction, which could affect islet survival due to inflammation [37].

The interaction between COVID-19 and DM could also be bidirectional. SARS-CoV-2 has been observed to predispose new-onset DM and vice versa DM comorbidities might worsen COVID-9 severity [34,37]. In normal people, the expression of ACE2 in the pancreas was even higher than in the lungs. The part of the pancreas which expresses ACE2 is the exocrine glands and islets [33]. In new-onset diabetes cases, SARS-CoV-2 might bind ACE2 and/ or Neuropilin 1 (NRP1) receptors in pancreatic $\beta$-cells through infected endothelium, thus damaging the pancreas which leads to altered insulin production [34,35]. After SARS-CoV2 was replicated inside the cell, JAK/STAT, PAK1/2, JNK1/2, EIF2a pathways were then activated and induced upregulation of stress and inflammation [35]. The identity of $\beta$-cells was lost due to cellular dedifferentiation, which leads to upregulation of cellular stress and apoptosis [35,38]. It induces cellular dysfunction that could decrease insulin secretion; hence hyperglycemia was observed [30].

\section{Post-COVID-19 syndrome}

Post-COVID-19 syndrome is a continuity of symptoms in three weeks or higher after the diagnosis. Around 10\% of COVID-19 patients experienced post-COVID syndrome and this was not only about severe acute patients but also all categories [1]. Post-COVID-19 syndrome has been related to various organ systems i.e., such as pulmonary, cardio-hematology, neuropsychiatry, renal, endocrine, gastrointestinal, and dermatology [39].

\subsection{Pulmonary manifestations}

There were several pulmonary manifestations from COVID-19 varying from dyspnea to fibrotic lung damage. It was estimated that there were 42-66\% of COVID-19 patients had dyspnea as persistent symptoms after experiencing COVID-19 [40]. Research conducted by Zhao et al showed that there were 70.91\% of COVID-19 patients evaluated by high-resolution computed tomography (HRCT) had radiological abnormalities after three months of discharge. In the context of respiratory function, a spirometry test was done on these patients. Lung function abnormalities were observed in $25.45 \%$ of these patients [41].

\subsection{Cardio-hematology manifestations}

There were up to $20 \%$ of COVID-19 survivors who experienced chest pain [40]. Ongoing myocardial inflammation showed during magnetic resonance imaging (MRI) in about $60 \%$ after two months of COVID19 cases [42]. Cardiovascular sequelae can manifest due to direct viral tropism, ACE2 downregulation, and immunologic response affecting the myocardial, pericardial, and conduction systems of the heart [40]. There 
were about $2.5 \%$ of COVID-19 survivors in total who experienced a segmental pulmonary embolism, intracardiac thrombus, thrombosed arteriovenous fistula, and ischemic stroke [43]. Coagulopathy related to COVID-19 occurred due to hyperinflammatory and hypercoagulable state consistently [40].

\subsection{Neuropsychiatric manifestations}

Post-viral syndrome consisting of depression and anxiety was observed in about $30-40 \%$ of patients who survived from COVID-19. Neurologic manifestations like migraine-like headaches, late-onset-headaches, persistent loss of taste and smell, and cognitive impairment also shown from patients following COVID-19. Viral tropism, severe systemic inflammation including neurodegeneration, and microvascular thrombosis attributed to the occurrence of neuropsychiatric abnormalities in post-COVID-19 infection [40].

\subsection{Renal manifestations}

SARS-CoV-2 probably affects renal cells directly because of its characteristics and physiology [44]. Target infections of SARS-CoV-2 are ACE2 and TMPRSS2 which are localized in the proximal tubules and collecting duct [45]. Another mechanism of renal injury was explained by the immune response which caused a cascade of renal injury i.e., acute tubular injury, interstitial inflammation, microangiopathy, proteinuria, and glomerulopathy [44].

\subsection{Endocrine manifestations}

COVID-19 possibly causes endocrine dysfunction which potentially affects multi-system disease. This characteristic output is insufficiency of adrenal, low T3 and TSH syndrome, and hyperprolactinemia [46]. In central hypopituitarism, molecular mimicry between SARS viral proteins and ACTH induced anti-ACTH antibodies with result destruction of ACTH positive cells [47]. Glucose metabolism was also disturbed because of morphological, transcriptional, and $\beta$-cells functional changes [48]. Early symptoms for glucose problems specially new-onset was DKA or hyperosmolarity [49].

\subsection{Gastrointestinal (GI) manifestations}

Post COVID-19 patients commonly present GI symptoms such as diarrhea, abdominal pain, and nausea or vomiting [50]. The prevalence of GI symptoms in one month or more after COVID-19 is ranging around $16 \%$ until $40 \%$. However, these numbers were much closer to the former than the latter percentage [51]. The GI symptoms from patients might be related to psychological factors like post-traumatic stress disorder, disruption of the gut microbiome, or continual intestinal inflammation [52].

\subsection{Dermatology manifestations}

Skin rash was observed in 3\% of COVID-19 survivors at six months follow-up [53]. It was estimated that $20 \%$ of the patients experienced hair loss caused by a viral infection or a stress response following COVID-19 experience [40].

\section{Hyperbaric oxygen therapy: COVID-19}

In a study conducted by Thibodeaux et al., patients were reported to recover from breathing difficulties after one session of HBOT. An average of five sessions of HBOT has been recommended for patients with 
50\% reduction in $\mathrm{FiO}_{2}$ [54]. Hypoxia and other abnormalities in COVID-19 could be treated by HBOT. Improvements in the patient's condition were observed by elevation in blood oxygen saturation and lung inflammation reduction as observed by CT scans [55]. In long COVID-19 cases, HBOT was statistically and clinically significant to improve both the overall fatigue score and a range of cognitive domains using validated scales [56].

HBOT has been declared to ameliorate altered mitochondrial function by balancing mitochondrial respiration and glycolysis possibly via opposing cellular caloristasis networks of viral infection [57]. HBOT has the capability to reduce the inflammatory response [58]. The process of HBOT consists of increasing: 1) oxygen dissolved in the alveolar and inflammatory barrier, 2) the diffusion rate and distance of oxygen, 3) the dissolution of oxygen in blood plasma and oxygen saturation of hemoglobin, and 4) the delivery of oxygen [14].

\section{Hyperbaric oxygen therapy on new-onset diabetes mellitus of post-COVID 19 syndrome: mechanism of action}

Several pathological alterations have been found in the COVID-19 patients both locally in the pancreas and systematically as discussed in the previous sections. Similar to T1DM, new-onset diabetes in COVID-19 patients has been associated with pancreatic injury due to SARS-CoV-2 infection, which induces $\beta$-cell apoptosis and decreases insulin secretion $[33,59,60]$. HBOT works by increasing oxygen partial pressure, wherein has been related to better cellular outcomes. Hyperoxia increases reactive oxygen species (ROS) which is mediated by NADPH oxidase enzyme and respiratory chain in mitochondria [9,57]. In acute therapy, the activity of mitochondria is decreased as compensation for ROS elevation, thus reducing ROS production on its own. In the latter condition, increased endogenous antioxidant levels were shown i.e., glutathione (GSH), glutamate peroxidase (GPx), and glutamate reductase (GR) [20,57]. HBOT was correlated with increased bcl-2, an anti-apoptotic marker, and bax depletion, a pro-apoptotic marker [21]. In the case of acute pancreatitis given HBOT, increase in $\beta$-cell proliferation level was found [61-63]. Local pancreatic inflammation also has been related to T1DM, which alters cellular signaling and decreases cellular survival. $\mathrm{Yu}$ et al showed HBOT attenuates pancreatic inflammation through NF- $\mathrm{KB}$ pathway [64]. Increases in pancreatic $\beta / \alpha$ cells ratio have been found after HBOT [65]. These conditions might result in improved cellular survival, which in turn, restores the function of the pancreas in maintaining glucose homeostasis.

SARS-CoV-2 enters the cell through ACE2 or TMPRSS22, which is expressed in various organs including the pancreas and its microvasculature [60]. These receptors might be a potential pathway for structural and functional microvascular transformation, hence leading to cytokine release, local inflammation, and tissue hypoxia. Hypoxia-inducible factor $1 \alpha$ (HIF-1 $\alpha$ ) possesses a critical role in organizing adaptive response during hypoxia, which was upregulated during SARS-CoV-2 infection. Tian et al showed SARS$\mathrm{CoV}-2$ ORF3a protein induces damage in mitochondria to promote HIF-1 $\alpha$ production. HIF-1 $\alpha$ was also found to play a role in facilitating SARS-CoV-2 infections [66]. On the other hand, HBOT could decrease HIF-1 $\alpha$ expression and modulate both its activation and stability [67,68]. Although the role of HIF-1 $\alpha$ in generating post-COVID-19 syndrome is not clear yet, those processes might benefit in preventing worse outcomes considering reinfection might occur. HBOT also reverses these conditions by stimulating vasoconstriction at an adequate oxygen environment, in combination with upregulation of vascular endothelial growth factor (VEGF) and its mRNA expression [69]. Stimulation of angiogenesis after HBOT also contributes to improved vascularization and oxygen delivery [69-71].

Hyperglycemia state has been linked to promoting nitric oxide (NO) production via the expression of iNOS and eNOS. A meta-analysis conducted by Assman et al showed that NO level was increased in T1DM 
patients of European ethnicity [72]. The resultant effects of NO were concentration-dependent, which could be beneficial or detrimental. NO has vasodilation properties that could reduce hypertension. However, in the greater levels, NO might react with $\mathrm{O}_{2}^{-}$which produces more free radicals such as peroxynitrite, thus stimulating lipid peroxidation, metabolism of arachidonic acid, and prostanoid production [73]. Impaired endothelial function was seen in this case as the result. Resanovic et al showed patients undergoing HBOT at 2.4 ATA/1h for 10 sessions decreased in iNOS activity, which catalyzes NO production [74]. Depletion of creactive protein (CRP) and free fatty acids (FFA) levels indicates improvement of chronic inflammation in diabetic patients. Systemic inflammation was also suppressed due to the expression of anti-inflammatory cytokines such as IL-10, thus enhancing the immune system. These conditions decrease the phosphorylation of ERK1/2 and Akt, which in turn decreases activation of NF- $\kappa$ B. As the result, iNOS activity was downregulated [74]. It is important to note that NO levels differ between individuals depending on existing comorbidities. Gurdol et al reported increased NO levels in patients with diabetic foot ulcers who underwent HBOT 2.4 ATA/3x25 minute/day for 6 days was associated with improvement in wound healing [75]. Increase in NO level was also associated with enhanced phagocytic activity [76]. These results might reflect the role of HBOT in modulating NO levels.

Despite restoring insulin production, improvement in insulin sensitivity of T2DM patients was also seen after HBOT [77-79]. This effect was mediated by activation of the insulin signaling pathway in the muscles, notably IRS1-Akt-GLUT4. Phosphorylation of Akt protein will promote GLUT4 expression, thus decreasing blood glucose levels. AMPK activation will enhance fatty acid oxidation and glucose utilization via adiponectin, thus fulfilling cellular energy demand [79]. Hepatic glycogen storage increased concomitantly by reducing GLUT2 transportation to the membrane [65]. Cellular metabolism was shifted into glycolysis, which preserves higher levels of ATP and enhances lactate clearance [80]. Elevated expression of uncoupling protein-1 (UCP1) in brown adipose tissue (BAT), as well as increased cells density, also play a role in UCP1mediated thermogenesis, by which these processes were dependent on glucose utilization and independent of fatty acid oxidation [79,81]. This phenomenon implied HBOT also exerts anti-atherogenic effects. These processes might benefit in alleviating fatigue amongst post-COVID-19 syndrome patients, in which fatty acid oxidation decreased and lactate accumulation increased [82]. Hence, HBOT could be proposed as one of the treatment modalities for patients with pre-existing DM comorbidity as well.

COVID-19 has been known to affect multiple organs both directly and indirectly. Surprisingly, few symptoms presented as post-COVID-19 syndrome i.e., fatigue, muscular weakness, joint pain, dyspnea, cough, anxiety, depression, brain fog, palpitations, chest pain, thromboembolism, chronic kidney disease, and hair loss [40]. HBOT has been known to have beneficial effects on organ systems until biomolecular levels. Few studies have been conducted to elucidate HBOT effects in COVID-19/post-COVID-19 patients. Robbins et al showed improvement in executive function, attention, verbal function, information processing, global cognition, and chalder fatigue scale amongst long COVID-19 patients receiving HBOT at 2.4 ATA/105 min for 10 sessions [56]. Experimental studies also showed that 2.5 ATA/90 min HBO exposure for 14 days could attenuate pulmonary fibrosis induced by bleomycin in vivo and in vitro via TGF- $\beta$-induced fibroblast activation downregulation [83]. It was observed HBOT also could modulate NO expression, wherein disease with low NO levels, HBOT might upregulate it [75]. Akaberi et al reported NO exerts antiviral effects to inhibit SARS-CoV-2 replication in vitro, thus preventing viral cytopathic effects [84].

\section{Safety of hyperbaric oxygen therapy}

Hyperbaric oxygen therapy is generally safe at a particular dose. However, attention should be paid to all patients receiving HBOT, especially those with relative contraindications i.e., claustrophobia, upper respiratory tract infection, etc. Whilst absolute contraindication is untreated pneumothorax [85]. Side effects 
commonly occur due to high pressure and high oxygen concentration administration. Most often, therapy above 4 ATA results in barotrauma and oxygen toxicity. Although serious complications rarely occur, safety rules must be followed by all health workers involved in HBOT $[85,86]$.

\section{Conclusion}

Hyperbaric oxygen therapy might be a potential therapy for improving new-onset DM among postCOVID-19 syndrome patients. These effects might be attributed to improvement in cellular function, especially pancreatic $\beta$-cells which restores insulin production concomitantly with the elevation of glucose utilization and fatty acid oxidation. Modulation of inflammatory pathway and increase in lactate clearance might improve other post-COVID-19 syndrome alterations. These findings must be taken into consideration since post-COVID-19 syndrome incidence tends to rise as pandemic still evolves. However, lack of literature studying these effects on new-onset DM among post-COVID-19 syndrome patients should be noted. Further studies regarding biomolecular, structural, and functional changes in pancreatic $\beta$-cells correlated with clinical improvement are required in order to understand the mechanism comprehensively. Therapeutic dose, duration, and side effects also should be studied to achieve optimal effectivity.

\section{Acknowledgements}

We would like to thank all the authors for their contribution to paper writings.

\section{References}

[1] Pavli A, Theodoridou M, Maltezou HC. Post-COVID Syndrome: Incidence, Clinical Spectrum, and Challenges for Primary Healthcare Professionals. Archives of Medical Research. 2021;52(6):575.

[2] Robinson SM, Rasch S, Beer S, Valantiene I, Mickevicius A, Schlaipfer E, et al. Systemic Inflammation Contributes to Impairment of Quality of Life in Chronic Pancreatitis. Scientific Reports 2019 9:1. 2019;9(1):1-8.

[3] Gupta A, Madhavan M v., Sehgal K, Nair N, Mahajan S, Sehrawat TS, et al. Extrapulmonary Manifestations of COVID-19. Nature Medicine. 2020;26(7):1017-32.

[4] Hamming I, Timens W, Bulthuis MLC, Lely AT, Navis GJ, van Goor H. Tissue Distribution of ACE2 Protein, the Functional Receptor for SARS Coronavirus. A First Step in Understanding SARS Pathogenesis. The Journal of Pathology. 2004;203(2):631-7.

[5] Azer SA. COVID-19: Pathophysiology, Diagnosis, Complications and Investigational Therapeutics. New Microbes and New Infections. 2020;37:100738.

[6] Fignani D, Licata G, Brusco N, Nigi L, Grieco GE, Marselli L, et al. SARS-CoV-2 Receptor Angiotensin I-Converting Enzyme Type 2 (ACE2) is Expressed in Human Pancreatic $\beta$-Cells and in the Human Pancreas Microvasculature. Frontiers in Endocrinology. 2020 Nov 13;11:876.

[7] WHO. WHO Coronavirus (COVID-19) Dashboard | WHO Coronavirus (COVID-19) Dashboard with Vaccination Data [Internet]. 2021 [cited 2021 Dec 30]. Available from: https://covid19.who.int/

[8] Shah J. Hyperbaric Oxygen Therapy. The Journal of the American College of Certified Wound Specialists. 2010;2(1):9.

[9] Poff AM, Kernagis D, D’Agostino DP. Hyperbaric Environment: Oxygen and Cellular Damage Versus Protection. Comprehensive Physiology. 2017 Jan 1;7(1):213-34.

[10] Paganini M, Bosco G, Perozzo FAG, Kohlscheen E, Sonda R, Bassetto F, et al. The Role of Hyperbaric Oxygen Treatment for COVID-19: A Review. Advances in Experimental Medicine and Biology. 2021;1289:27-35.

[11] Baitule S, Patel AH, Murthy N, Sankar S, Kyrou I, Ali A, et al. A Systematic Review to Assess the Impact of Hyperbaric Oxygen Therapy on Glycaemia in People with Diabetes Mellitus. Medicina (Kaunas, Lithuania). 2021;57(10).

[12] Vera-Cruz P, Guerreiro F, Ribeiro MJ, Guarino MP, Conde S v. Hyperbaric Oxygen Therapy Improves Glucose Homeostasis in Type 2 Diabetes Patients: A Likely Involvement of the Carotid Bodies. Advances in Experimental Medicine and Biology. 2015;860:221-5.

[13] Faleo G, Fotino C, Bocca N, Molano RD, Zahr-Akrawi E, Molina J, et al. Prevention of Autoimmune Diabetes and Induction of $\beta$-Cell Proliferation in NOD Mice by Hyperbaric Oxygen Therapy. Diabetes. 2012 Jul;61(7):1769-78.

[14] Harch P. Hyperbaric Oxygen Treatment of Novel Coronavirus (COVID-19) Respiratory Failure. Medical Gas Research. 
2020;10(2):61.

[15] Pitkin A, Davies N. Hyperbaric Oxygen Therapy. British Journal of Anaesthesia. 2001;1:150-6.

[16] Hajhosseini B, Kuehlmann BA, Bonham CA, Kamperman KJ, Gurtner GC. Hyperbaric Oxygen Therapy: Descriptive Review of the Technology and Current Application in Chronic Wounds. Plastic and Reconstructive Surgery Global Open. 2020;8(9).

[17] Zhou Q, Huang G, Yu X, Xu W. A Novel Approach to Estimate ROS Origination by Hyperbaric Oxygen Exposure, Targeted Probes and Specific Inhibitors. Cellular Physiology and Biochemistry. 2018;47(5):1800-8.

[18] Thom SR. The Physiology and Pathophysiology of the Hyperbaric and Diving Environments: Oxidative stress is fundamental to Hyperbaric Oxygen Therapy. Journal of Applied Physiology. 2009;106(3):988.

[19] Zarkovic N. Roles and Functions of ROS and RNS in Cellular Physiology and Pathology. Cells. 2020 Mar 21;9(3).

[20] Chang HC, Yang YR, Wang RY. Effects of Repetitive Hyperbaric Oxygen Therapy on Neuroprotection in Middle Cerebral Artery Occlusion Rats. Brain Research. 2020 Dec 1;1748.

[21] Gottfried I, Schottlender N, Ashery U. Hyperbaric Oxygen Treatment-from Mechanisms to Cognitive Improvement. Biomolecules. 2021;11(10).

[22] Lipsky BA, Berendt AR. Hyperbaric Oxygen Therapy for Diabetic Foot Wounds: Has Hope Hurdled Hype? Diabetes Care. 2010;33(5):1143.

[23] Alexiadou K, Doupis J. Management of Diabetic Foot Ulcers. Diabetes Therapy. 2012;3(1):1-15.

[24] Ekanayake L, Doolette DJ. Effects of Hyperbaric Oxygen Treatment on Blood Sugar Levels and Insulin Levels in Diabetics. South Pac Underw Med Soc J. 2001;31:16-20.

[25] Trytko B, Bennett M. Blood Sugar Changes in Diabetic Patients Undergoing Hyperbaric Oxygen Therapy. SPUMS J. 2003; 33(2); 62-69).

[26] Al-Waili NS, Butler GJ, Beale J, Abdullah MS, Finkelstein M, Merrow M, et al. Influences of Hyperbaric Oxygen on Blood Pressure, Heart Rate and Blood Glucose Levels in Patients with Diabetes Mellitus and Hypertension. Archives of Medical Research. 2006;37(8):991-7.

[27] Peleg RK, Fishlev G, Bechor Y, Bergan J, Friedman M, Koren S, et al. Effects of Hyperbaric Oxygen on Blood Glucose Levels in Patients with Diabetes Mellitus, Stroke or Traumatic Brain Injury and Healthy Volunteers: A Prospective, Crossover, Controlled Trial. Diving Hyperb Med. 2013 Dec;43(4):218-21.

[28] Xu Q, Wei YT, Fan SB, Wang L, Zhou XP. Repetitive Hyperbaric Oxygen Treatment Increases Insulin Sensitivity in Diabetes Patients with Acute Intracerebral Hemorrhage. Neuropsychiatric Disease and Treatment. 2017;13:421-6.

[29] Iii MH, Wojcik SM, Swaby J, Boes T. Blood Glucose Levels in Diabetic Patients Undergoing Hyperbaric Oxygen Therapy. Undersea and Hyperbaric Medical Society. 2019;46(4):437-45.

[30] Lima-Martínez, MM, Carrera Boada, C., Madera-Silva, MD, Marín, W., \& Contreras M. COVID-19 and Diabetes: A Bidirectional Relationship. Clinic and Research in Arteriosclerosis: Official Publication of the Spanish Arteriosclerosis Society. 2021;33(3):151-7.

[31] Hoffmann M, Kleine-Weber H, Schroeder S, Krüger N, Herrler T, Erichsen S, et al. SARS-CoV-2 Cell Entry Depends on ACE2 and TMPRSS2 and Is Blocked by a Clinically Proven Protease Inhibitor. Cell. 2020;181(2):271-280.e8.

[32] South AM, Diz DI, Chappell MC. COVID-19; ACE2; and the Cardiovascular Consequences. American Journal of Physiology Heart and Circulatory Physiology. 2020;318(5):H1084-H1090.

[33] Liu F, Long X, Zhang B, Zhang W, Chen X, Zhang Z. ACE2 Expression in Pancreas May Cause Pancreatic Damage After SARS-CoV-2 Infection. Clinical Gastroenterology and Hepatology. 2020;18(9):2128-2130.e2.

[34] Maddaloni E, Buzzetti R. COVID-19 and Diabetes Mellitus: Unveiling the Interaction of Two Pandemics. Diabetes/Metabolism Research and Reviews. 2020;36(7):19-20.

[35] Memon B, Abdelaim EM. ACE2 Function in the Pancreatic Islet: Implications for Relationship Between SARS-Cov-2 and Diabetes. Acta Physiologica. 2021;233(4):e13733.

[36] Aloysius MM, Thatti A, Gupta A, Sharma N, Bansal P, Goyal H. COVID-19 Presenting as Acute Pancreatitis. Pancreatology. 2020;20(5):1026-7.

[37] Azar WS, Njeim R, Fares AH, Azar NS, Azar ST, el Sayed M, et al. COVID-19 and Diabetes Mellitus: How One Pandemic Worsens the Other. Reviews in Endocrine and Metabolic Disorders. 2020;21(4):451-63.

[38] Sathish T, Kapoor N, Cao Y, Tapp RJ, Zimmet P. Proportion of Newly Diagnosed Diabetes In COVID-19 Patients: A Systematic Review and Meta-Analysis. Diabetes, Obesity and Metabolism. 2021;23(3):870-4.

[39] Tirelli U, Taibi R, Chirumbolo S. Post COVID Syndrome: A New Challenge for Medicine. European Review for Medical and Pharmacological Sciences. 2021;25(12):4422-5.

[40] Nalbandian A, Sehgal K, Gupta A, Madhavan M v., McGroder C, Stevens JS, et al. Post-Acute COVID-19 Syndrome. Nature Medicine. 2021;27(4):601-15.

[41] Zhao Y miao, Shang Y min, Song W bin, Li Q quan, Xie H, Xu Q fu, et al. Follow-Up Study of The Pulmonary Function and Related Physiological Characteristics of COVID-19 Survivors Three Months After Recovery. E-ClinicalMedicine. $2020 ; 25$.

[42] Puntmann VO, Carerj ML, Wieters I, Fahim M, Arendt C, Hoffmann J, et al. Outcomes of Cardiovascular Magnetic Resonance Imaging in Patients Recently Recovered from Coronavirus Disease 2019 (COVID-19). JAMA Cardiology. 2020;5(11):1265-73.

[43] Patell R, Bogue T, Koshy A, Bindal P, Merrill M, Aird WC, et al. Postdischarge Thrombosis and Hemorrhage in Patients with COVID-19. Blood. 2020;136(11):1342-6.

[44] Pramod S, Kheetan M, Ogu I, Alsanani A, Khitan Z. Viral Nephropathies, Adding SARS-Cov-2 to the List. International 
Journal of Nephrology and Renovascular Disease. 2021;14:157-64.

[45] Park S. Epidemiology; Virology; and Clinical Features of Severe Acute Respiratory Syndrome -Coronavirus-2 (SARS-CoV-2; Coronavirus Disease-19). Clin Exp Pediatr. 2020;63(4):119-24.

[46] Kumar B, Gopalakrishnan M, Garg MK, Purohit P, Banerjee M, Sharma P, et al. Endocrine Dysfunction among Patients with COVID-19: A Single-center Experience from a Tertiary Hospital in India. Indian Journal of Endocrinology and Metabolism. 2021;25(1):14-9.

[47] Wei L, Sun S, Zhang J, Zhu H, Xu Y, Ma Q, et al. Endocrine Cells of the Adenohypophysis in Severe Acute Respiratory Syndrome (SARS). Biochemistry and Cell Biology. 2010;88(4):723-30.

[48] Kazakou P, Paschou SA, Psaltopoulou T, Gavriatopoulou M, Korompoki E, Stefanaki K, et al. Early and Late Endocrine Complications of COVID-19. Endocrine Connections. 2021;10(9):R229-39.

[49] Rubino F, Amiel SA, Zimmet P, Alberti G, Bornstein S, Eckel RH, et al. New-Onset Diabetes in COVID-19. New England Journal of Medicine. 2020;383(8):789-90.

[50] Redd WD et al. Prevalence and Characteristics of Gastrointestinal Symptoms in Patients with Severe Acute Respiratory Syndrome Coronavirus 2 Infection in the United States: A Multicenter Cohort Study. Gastroenterology. 2020;159(2):765-7.

[51] Blackett JW, Li J, Jodorkovsky D, Freedberg DE. Prevalence and Risk Factors for Gastrointestinal Symptoms After Recovery from COVID-19. Neurogastroenterology and Motility. 2021;(August):1-9.

[52] Schmulson, M; Ghoshal, UC; Barbara G. Managing the Inevitable Surge of Post-COVID-19 Functional Gastrointestinal Disorders. Am J Gastroenterol. 2021;116(1):4-7.

[53] Huang C, Huang L, Wang Y, Li X, Ren L, Gu X, et al. 6-Month Consequences of COVID-19 in Patients Discharged from Hospital: A Cohort Study. Lancet. 2021;397(10270):220-32.

[54] Thibodeaux K, Speyrer M, Raza A, Yaakov R, Serena TE. Hyperbaric Oxygen Therapy in Preventing Mechanical Ventilation in COVID-19 Patients: A Retrospective Case Series. Journal of Wound Care. 2020;29:S4-8.

[55] de Maio A, Hightower LE. COVID-19, Acute Respiratory Distress Syndrome (ARDS), and Hyperbaric Oxygen Therapy (HBOT): What Is The Link?. Cell Stress and Chaperones. 2020;25(5):717-20.

[56] Robbins T, Gonevski M, Clark C, Baitule S, Sharma K, Magar A, et al. Hyperbaric Oxygen Therapy for the Treatment of Long COVID: Early Evaluation of A Highly Promising Intervention. Clinical Medicine. 2021;21(6):e629-32.

[57] Tezgin D, Giardina C, Perdrizet GA, Hightower LE. The Effect of Hyperbaric Oxygen on Mitochondrial and Glycolytic Energy Metabolism: The Caloristasis Concept. Cell Stress and Chaperones. 2020;25(4):667-77.

[58] Halbach JL, Prieto JM, Wang AW, Hawisher D, Cauvi DM, Reyes T, et al. Early Hyperbaric Oxygen Therapy Improves Survival in A Model of Severe Sepsis. American Journal of Physiology - Regulatory Integrative and Comparative Physiology. 2019;317(1):R160-8.

[59] Geravandi S, Mahmoudi-aznaveh A, Azizi Z, Maedler K, Ardestani A. SARS-Cov-2 and Pancreas: A Potential Pathological Interaction?. Trends in Endocrinology and Metabolism. 2021;32(11):842-5.

[60] Müller JA, Groß R, Conzelmann C, Krüger J, Merle U, Steinhart J, et al. SARS-CoV-2 Infects and Replicates in Cells of the Human Endocrine and Exocrine Pancreas. Nature Metabolism. 2021;3(2):149-65.

[61] Christophi C, Millar I, Nikfarjam M, Muralidharan V, Malcontenti-Wilson C. Hyperbaric Oxygen Therapy for Severe Acute Pancreatitis. Journal of Gastroenterology and Hepatology. 2007;22(11):2042-6.

[62] Festugato M, Coelho CP, Fiedler G, Machado FP, Gonçalves MC, Bassani FR, et al. Hyperbaric Oxygen Therapy Effects on Tissue Lesions in Acute Pancreatitis. Experimental study in rats. Journal of the Pancreas. 2008;9(3):275-82.

[63] Koh SL, Tan JW, Muralidharan V, Christophi C. The Effect of Hyperbaric Oxygen on Apoptosis and Proliferation in Severe Acute Pancreatitis. Hpb. 2009;11(8):629-37.

[64] Yu X, Li YG, He XW, Li XR, Din BN, Gan Y, et al. Hyperbaric Oxygen Reduces Inflammatory Response in Acute Pancreatitis by Inhibiting NF-Kb Activation. European Surgical Research. 2009;42(2):130-5.

[65] SONG L, YUAN J, LIU Y, ZHANG D, ZHANG C, LIN Q, et al. Ghrelin System is Involved in Improvements in Glucose Metabolism Mediated by Hyperbaric Oxygen Treatment in A Streptozotocin-Induced Type 1 Diabetes Mouse Model. Molecular Medicine Reports. 2020;22(5):3767-76.

[66] Tian M, Liu W, Li X, Zhao P, Shereen MA, Zhu C, et al. HIF-1 $\alpha$ Promotes SARS-Cov-2 Infection and Aggravates Inflammatory Responses to COVID-19. Signal Transduction and Targeted Therapy. 2021;6(1):1-13.

[67] Li Y, Zhou C, Calvert JW, Colohan ART, Zhang JH. Multiple Effects of Hyperbaric Oxygen on the Expression of HIF-1 $\alpha$ and Apoptotic Genes in A Global Ischemia-Hypotension Rat Model. Experimental Neurology. 2005;191(1):198-210.

[68] Sunkari VG, Lind F, Botusan IR, Kashif A, Liu ZJ, Ylä-Herttuala S, et al. Hyperbaric Oxygen Therapy Activates HypoxiaInducible Factor 1 (HIF-1), Which Contributes to Improved Wound Healing in Diabetic Mice. Wound Repair and Regeneration. 2015;23(1):98-103.

[69] Oley MH, Oley MC, Noersasongko AD, Hatta M, Philips GG, Agustine, et al. Effects of Hyperbaric Oxygen Therapy on Vascular Endothelial Growth Factor Protein and mRNA in Crush Injury Patients: A Randomized Controlled Trial Study. International Journal of Surgery Open. 2021;29:33-9.

[70] Cuthbertson CM, Christophi C. Potential Effects of Hyperbaric Oxygen Therapy in Acute Pancreatitis. ANZ Journal of Surgery. 2006;76(7):625-30.

[71] Peña-Villalobos I, Casanova-Maldonado I, Lois P, Prieto C, Pizarro C, Lattus J, et al. Hyperbaric Oxygen Increases Stem Cell Proliferation, Angiogenesis and Wound-Healing Ability of WJ-Mscs in Diabetic Mice. Frontiers in Physiology. 2018;9(July):1-15. 
[72] Assmann TS, Brondani LA, Bouças AP, Rheinheimer J, de Souza BM, Canani LH, et al. Nitric Oxide Levels in Patients with Diabetes Mellitus: A Systematic Review and Meta-Analysis. Nitric Oxide - Biology and Chemistry. 2016;61:1-9.

[73] Onozato ML, Tojo A, Goto A, Fujita T, Wilcox CS. Oxidative Stress and Nitric Oxide Synthase in Rat Diabetic Nephropathy: Effects Of ACEI And ARB. Kidney International. 2002;61(1):186-94.

[74] Resanovic I, Gluvic Z, Zaric B, Sudar-Milovanovic E, Jovanovic A, Milacic D, et al. Early Effects of Hyperbaric Oxygen on Inducible Nitric Oxide Synthase Activity/Expression in Lymphocytes Of Type 1 Diabetes Patients: A Prospective Pilot Study. International Journal of Endocrinology. 2019;2019.

[75] Gurdol F, Cimsit M, Oner-Iyidogan Y, Kocak H, Sengun S, Yalcinkaya-Demirsoz S. Collagen Synthesis, Nitric Oxide and Asymmetric Dimethylarginine in Diabetic Subjects Undergoing Hyperbaric Oxygen Therapy. Physiological Research. 2010;59(3):423-9.

[76] Memar MY, Yekani M, Alizadeh N, Baghi HB. Hyperbaric oxygen therapy: Antimicrobial Mechanisms and Clinical Application for Infections. Biomedicine and Pharmacotherapy. 2019;109:440-7.

[77] Wilkinson D, Chapman IM, Heilbronn LK. Hyperbaric Oxygen Therapy Improves Peripheral Insulin Sensitivity in Humans. Diabetic Medicine. 2012;29(8):986-9.

[78] Wilkinson DC, Chapman IM, Heilbronn LK. Hyperbaric Oxygen but Not Hyperbaric Air Increases Insulin Sensitivity in Men with Type 2 Diabetes Mellitus. Diving and Hyperbaric Medicine. 2020;50(4):386-90.

[79] Liu Y, Zhang D, Yuan J, Song L, Zhang C, Lin Q, et al. Hyperbaric Oxygen Ameliorates Insulin Sensitivity by Increasing GLUT4 Expression in Skeletal Muscle and Stimulating UCP1 in Brown Adipose Tissue in T2DM Mice. Frontiers in Endocrinology. 2020;11(January):1-11.

[80] Bosco G, Yang ZJ, Nandi J, Wang J, Chen C, Camporesi EM. Effects of Hyperbaric Oxygen on Glucose, Lactate, Glycerol and Anti-Oxidant Enzymes in the Skeletal Muscle of Rats During Ischaemia and Reperfusion. Clinical and Experimental Pharmacology and Physiology. 2007;34(1-2):70-6.

[81] Okamatsu-Ogura Y, Kuroda M, Tsutsumi R, Tsubota A, Saito M, Kimura K, et al. UCP1-Dependent and UCP1-Independent Metabolic Changes Induced by Acute Cold Exposure in Brown Adipose Tissue of Mice. Metabolism: Clinical and Experimental. 2020;113:154396.

[82] de Boer E, Petrache I, Goldstein NM, Olin JT, Keith RC, Modena B, et al. Decreased Fatty Oxidation and Altered Lactate Production During Exercise in Post-Acute COVID-19 Patients. American Journal of Respiratory and Critical Care Medicine. 2021;0:1-14.

[83] Yuan Y, Li Y, Qiao G, Zhou Y, Xu Z, Hill C, et al. Hyperbaric Oxygen Ameliorates Bleomycin-Induced Pulmonary Fibrosis in Mice. Frontiers in Molecular Biosciences. 2021;8(June):1-11.

[84] Akaberi D, Krambrich J, Ling J, Luni C, Hedenstierna G, Järhult JD, et al. Mitigation of the Replication of SARS-Cov-2 by Nitric Oxide In Vitro. Redox Biology. 2020;37.

[85] Kranke P, Bennett MH, Martyn-St James M, Schnabel A, Debus SE, Weibel S. Hyperbaric Oxygen Therapy for Chronic Wounds. Cochrane Database of Systematic Reviews. 2015;2015(6):20-2.

[86] Heyboer M, Sharma D, Santiago W, McCulloch N. Hyperbaric Oxygen Therapy: Side Effects Defined and Quantified. Advances in Wound Care. 2017;6(6):210-24. 